\title{
On Simply Normal Numbers to Different Bases
}

\author{
Verónica Becher \\ Universidad de Buenos Aires \\ vbecher@dc.uba.ar
}

\author{
Yann Bugeaud \\ Université de Strasbourg \\ bugeaud@math.unistra.fr
}

\author{
Theodore A. Slaman \\ University of California Berkeley \\ slaman@math.berkeley.edu
}

August 29, 2013

\begin{abstract}
Let $s$ be an integer greater than or equal to 2 . A real number is simply normal to base $s$ if in its base- $s$ expansion every digit $0,1, \ldots, s-1$ occurs with the same frequency $1 / s$. Let $\mathcal{S}$ be the set of positive integers that are not perfect powers, hence $\mathcal{S}$ is the set $\{2,3,5,6,7,10,11, \ldots\}$. Let $M$ be a function from $\mathcal{S}$ to sets of positive integers such that, for each $s$ in $\mathcal{S}$, if $m$ is in $M(s)$ then each divisor of $m$ is in $M(s)$ and if $M(s)$ is infinite then it is equal to the set of all positive integers. These conditions on $M$ are necessary for there to be a real number which is simply normal to exactly the bases $s^{m}$ such that $s$ is in $\mathcal{S}$ and $m$ is in $M(s)$. We show these conditions are also sufficient and further establish that the set of real numbers that satisfy them has full Hausdorff dimension. This extends a result of W. M. Schmidt (1961/1962) on normal numbers to different bases.
\end{abstract}

\section{Contents}

1 Introduction

2 Lemmas

2.1 Residue equivalence . . . . . . . . . . . . . . . . . . . 4

2.2 Block equivalence . . . . . . . . . . . . . . . . . . . . . . 6

2.3 A lower bound for simple discrepancy in Cantor sets . . . . . . . . . . . 8

2.4 Exponential sums on Cantor sets . . . . . . . . . . . . . . . . 10

2.5 An upper bound for simple discrepancy in Cantor sets . . . . . . . . . . . . 14

2.6 Simple discrepancy and concatenation . . . . . . . . . . . . . . . 17

\section{Existence}

\section{Hausdorff dimension}

\section{Introduction}

In 1909 Émile Borel [2] introduced the notions of simple normality and of normality to an integer base. Let $s$ be an integer greater than or equal to 2 . A real number $x$ whose expansion in base $s$ is given by

$$
x=\lfloor x\rfloor+\sum_{j \geqslant 1} a_{j} s^{-j},
$$


where $a_{j} \in\{0,1, \ldots, s-1\}$ for $j \geqslant 1$, is said to be simply normal to base $s$ if every digit $d \in\{0,1, \ldots, s-1\}$ occurs in the sequence $\left(a_{j}\right)_{j \geqslant 1}$ with the same frequency $1 / s$. That is, for every such digit $d$,

$$
\lim _{n \rightarrow \infty} \frac{\#\left\{j: 1 \leqslant j \leqslant n \text { and } a_{j}=d\right\}}{n}=\frac{1}{s} .
$$

A number $x$ is said to be normal to base $s$ if it is simply normal to base $s^{k}$ for every integer $k \geqslant 1$ 丹

Borel established that almost all real numbers, with respect to the Lebesgue measure, are normal to every integer base greater than or equal to 2. Several equivalent definitions of normality are given in the monograph [3].

Are there numbers that are simply normal to arbitrarily different bases? This question was implicit in the literature and hitherto only partially answered. Recall that two positive integers are multiplicatively dependent when one is a rational power of the other. It is already known that for any given set of bases closed under multiplicative dependence there are uncountably many numbers that are simply normal to each base in the given set and not simply normal to any base in its complement. The historical trace of this result goes back first to a theorem of Maxfield [12] showing that normality to one base implies normality to another when the two are multiplicatively dependent. Then Schmidt [15], improving a result by Cassels [5] and his previous result [14], showed that for any set of bases closed under multiplicative dependence, the set of real numbers that are normal to every base in the given set but not normal to any base in its complement is uncountable. Lastly, Becher and Slaman [1] established the analogous theorem denying simple normality instead of normality. These results, however, do not settle the behavior of simple normality to bases within multiplicative-dependence equivalence classes.

Already in 1957, Long [11] proved that if a real number is simply normal to base $s^{m}$ for infinitely many exponents $m$, then it is normal to base $s$, hence simply normal to base $s^{m}$ for every positive integer $m$. A straightforward analysis, see [4, Lemma 4.3], shows that for any base $s$ and exponent $m$, simple normality to base $s^{m}$ implies simple normality to base $s$. Hertling [9] investigated the converse and concluded that simple normality to base $r$ implies simple normality to base $s$ if and only if $r$ is a power of $s$. This leaves open the question of whether for any given base $s$ there are numbers that are simply normal to bases $s^{m}$ for just finitely many positive integers $m$. In the following theorem we settle the characterization of simple normality to different bases and considerably extend Schmidt's result [15].

Theorem 1. Let $\mathcal{S}$ be the set of positive integers that are not perfect powers, hence $\mathcal{S}$ is the set $\{2,3,5,6,7,10,11, \ldots\}$. Let $M$ be a function from $\mathcal{S}$ to sets of positive integers such that, for each $s$ in $\mathcal{S}$, if $m$ is in $M(s)$ then each divisor of $m$ is in $M(s)$ and if $M(s)$ is infinite then it is equal to the set of all positive integers. There is a real number $x$ such that, for every integer $s$ in $\mathcal{S}$ and every positive integer $m, x$ is simply normal to base $s^{m}$ if and only if $m$ is in $M(s)$. Moreover, the set of real numbers $x$ that satisfy this condition has full Hausdorff dimension.

Observe that when $M(s)$ is empty the real number $x$ is not simply normal to base $s$.

The proof of Theorem 1 uses both combinatorial and analytic tools within a global construction. First, consider the restricted problem, for each base $s \in \mathcal{S}$, of ensuring simple normality of the real number $x$ to each of the finitely many numbers $s^{m}$ for $m \in M(s)$,

\footnotetext{
${ }^{\dagger}$ To be accurate, the latter definition is not the one originally given by Borel, but equivalent to it.
} 
ensuring simple normality for each of finitely many numbers $r$ which are multiplicatively independent to $s$, and ensuring a failure of simple normality for $s^{n}$, where $n$ does not divide any element of $M(s)$. We construct an appropriate Cantor set such that almost every element with respect to its uniform measure is simply normal to each base $s^{m}$, for $m \in M(s)$, and is not simply normal to base $s^{n}$. Then, we use Fourier analysis to prove that almost all elements in this set are simply normal to every base which is multiplicatively independent to $s$. The latter technique was used first by Cassels [5] to prove that almost all elements of the middle-third Cantor set (with respect to the Cantor measure) are normal to every base which is not a power of 3. It was independently used by Schmidt [14 to address every pair of multiplicatively independent integers, and then extended by Schmidt [15] and Pollington [13].

The main novelty in the proof of Theorem 1 is the determination of this appropriate Cantor set. When its elements are viewed in base $s^{m}$, for $m \in M(s)$, each digit should occur with expected frequency $1 / s^{m}$, and when viewed in base $s^{n}$ there should be a bias for some digits over others. As in Bugeaud's [4, Theorem 6.1] proof of Hertling's theorem, we work with base $s^{\ell}$, where $\ell$ is a large common multiple of $n$ and the elements of $M(s)$. Among the numbers less than $s^{\ell}$, we find one or two, depending on the parity of $s$, which are balanced when written in any of the bases $s^{m}$ (that is, all the digits in base $s^{m}$ appear with equal frequency), and which are unbalanced when written in base $s^{n}$. We obtain the appropriate Cantor set by working in base $s^{\ell}$ and omitting these one or two digits. It takes a rather interesting combinatorial argument in modular arithmetic to show that such numbers less than $s^{\ell}$ exist.

Given this solution to the restricted problem, we construct a nested sequence of intervals by recursion with a unique real number $x$ in their intersection. A step in the recursion involves staying in one of the above Cantor sets long enough so that a large initial segment of the expansion of $x$ to base $s^{n}$ has that Cantor set's bias while also ensuring that the frequency of each digit in the expansion of $x$ to any of the other bases $r$ being considered continues its convergence to $1 / r$, thus giving simple normality to base $r$. Every base to which $x$ is required to be simply normal is under consideration from some point on in the construction and every base to which $x$ is required not to be simply normal is acted upon infinitely often. In case the function $M$ is computable (which means that for each $s \in \mathcal{S}, M(s)$ can be constructed by finitary means), then so is $x$.

Regarding metric results, Pollington [13] established that, for any given set of bases closed under multiplicative dependence, the set of real numbers that are normal to every base in the given set but not normal to any base in its complement has full Hausdorff dimension. More recently, it is proved in [4, Theorem 6.1] that, for every integer $s$ greater than or equal to 2 and every coprime integers $m$ and $n$ with $n$ greater than or equal to 2 , the set of real numbers which are simply normal to base $s^{m}$ but not simply normal to base $s^{n}$ has full Hausdorff dimension. In fact, the proof applies in the more general case in which $n$ does not divide $m$. The last statement of Theorem 1 ensuring full Hausdorff dimension considerably extends both results.

Notation. We denote by $\mathcal{S}$ the set of positive integers that are not perfect powers, so $\mathcal{S}=\{2,3,5,6,7,10,11, \ldots\}$. A base is an integer greater than or equal to 2 . For a base $s$, let $B_{s}=\{0,1, \ldots, s-1\}$ denote the set of digits used to represent real numbers in base $s$. For a finite set $V$ of non-negative integers, we denote by $\mathcal{L}(\ell, V)$ the sequences $\left(v_{0}, \ldots, v_{\ell-1}\right)$ of $\ell$ many elements of $V$. We refer to such sequences as blocks and denote the length of a block $w$ by $|w|$. For $w \in \mathcal{L}(\ell, V)$, we denote by $(w ; m)$ the sequence of blocks of length $m$ whose 
concatenation is the largest prefix of $w$ whose length is a multiple of $m$. We use repeatedly the observation that, for a base $s$ and positive integers $\ell$ and $n$ such that $\ell$ is a multiple of $n$, a block of length $\ell$ on $B_{s}$ can be seen as a block of length $\ell / n$ on $B_{s^{n}}$. Furthermore, we sometimes identify the block $b_{0} \ldots b_{\ell-1}$ on $B_{s}$ with the integer $b_{0} s^{\ell-1}+\ldots+b_{\ell-2} s+b_{\ell-1}$. We use the convention that a set is finite if it is empty or it has finitely many elements.

\section{Lemmas}

We start with a collection of lemmas which deal with one single base $s$ and its powers. We may think of $s$ as an element of $\mathcal{S}$ but the lemmas apply to any integer base.

\subsection{Residue equivalence}

Definition. Let $X, Y$ be sets of non-negative integers and let $M$ be a set of positive integers. Then $X$ and $Y$ are residue equivalent for $M$ if and only if, for every $m$ in $M$ and every integer $r$ with $0 \leqslant r<m$, the sets $\{x: x \in X$ and $x \equiv r \bmod m\}$ and $\{y: y \in Y$ and $y \equiv r \bmod m\}$ have the same cardinality. When $M=\{m\}$ is a singleton, we say that $X$ and $Y$ are residue equivalent for $m$.

Instead of directly considering $M$ as a set of positive integers, we first consider $M$ as a collection of residue classes modulo $n$, with multiplicity.

Definition. A multiset $M$ of residues $\bmod n$ is fair if there is a positive integer $k$ such that $M$ is the multiset in which each integer between 1 and $n-1$ appears with multiplicity $k$.

Observe that in case $n$ is 1 , the only fair multiset $M$ of residues mod 1 is the empty set. For a fair multiset $M$, we consider the collection of sums of elements of $M$.

Definition. Let $n, z, v, k$ be positive integers. We denote by $p(n, z, v, k)$ the number of ways that $z$ can be written as a sum of $v$ elements from the multiset $\{1, \ldots, 1, \ldots, n-1, \ldots, n-1\}$ in which every integer between 1 and $n-1$ is repeated exactly $k$ times.

For example, a rapid check shows that $p(3,6,4,2)=1$ and $p(2,1,1, k)=k$ for $k \geqslant 1$. Let $\phi$ denote Euler's totient function: $\phi(n)$ counts the number of positive integers less than or equal to $n$ that are relatively prime to $n$.

The following combinatorial theorem, kindly communicated to us by Mark Haiman, is the key tool for the proof of Lemma 3 below.

Theorem 2 (Haiman [7]). For any $n$ and $k$ positive integers, we have

$$
\sum_{\substack{z: n \text { divides } z \\ v: v \text { even }}} p(n, z, v, k)-\sum_{\substack{z: n \text { divides } \\ v: v \text { odd }}} p(n, z, v, k)=n^{k-1} \phi(n) .
$$

Proof. The generating function for $p(n, z, v, k)$ is given by

$$
\sum_{s \geqslant 1, v \geqslant 1} p(n, z, v, k) x^{v} q^{s}=\prod_{j=1}^{n-1}\left(1+x q^{j}\right)^{k} .
$$


To calculate

$$
d(n, k)=\sum_{\substack{z: n \text { divides } z \\ v: v \text { even }}} p(n, z, v, k)-\sum_{\substack{z: n \text { divides } z \\ v: v \text { odd }}} p(n, z, v, k),
$$

set $x=-1$ and choose $q$ to be an $n$-th root of unity. Then, averaging over all the $n$-th roots of unity, we obtain

$$
d(n, k)=\frac{1}{n} \sum_{w^{n}=1} \prod_{j=1}^{n-1}\left(1-w^{j}\right)^{k} .
$$

If $w$ is not a primitive $n$-th root of unity, then $w^{j}=1$ for some positive integer $j$ less than $n$ and the above product vanishes. If $w$ is a primitive $n$-th root of unity, then the above product is equal to the $k$-th power of

$$
\prod_{j=1}^{n-1}\left(1-e^{2 \pi i j / n}\right)
$$

Setting $z=1$ in the equality

$$
\prod_{j=1}^{n-1}\left(z-e^{2 \pi i j / n}\right)=\left(z^{n}-1\right) /(z-1)=1+z+z^{2}+\ldots+z^{n-1},
$$

we get that

$$
\prod_{j=1}^{n-1}\left(1-e^{2 \pi i j / n}\right)=n .
$$

It then follows that $d(n, k)=n^{k-1} \phi(n)$.

The next lemma extends the following easy observation. Let $m_{1}, m_{2}$ and $n$ be positive integers such that $n$ does not divide $m_{1}$ nor $m_{2}$. Then, the sets $\left\{0, m_{1}+m_{2}\right\}$ and $\left\{m_{1}, m_{2}\right\}$ are residue equivalent for $m_{1}$ and for $m_{2}$, but not for $n$.

Lemma 3. Let $M$ be a non-empty finite set of positive integers and $n$ be a positive integer that does not divide any element of $M$. Then, there are sets $X$ and $Y$ of non-negative integers which are residue equivalent for $M$ and not residue equivalent for $n$.

Proof. Without loss of generality, we may assume that the multiset of residues of the elements of $M$ modulo $n$ is fair. If necessary, $M$ can be extended to a set with this property and proving the lemma for this larger set also verifies it for $M$.

Let $E(M)$ be the multiset of non-negative integers that can be expressed as sums of evenly many elements of $M$, where the multiplicity of each element is the number of ways that it can be expressed as such a sum. Here, we adopt the convention that the empty sum is even and has value 0 . Likewise, let $O(M)$ be the analogous multiset defined using sums of oddly many elements of $M$.

Write $M=\left\{m_{1}, m_{2}, \ldots, m_{k}\right\}$ and $M_{j}=\left\{m_{i}: i \leqslant j\right\}$ for $j=1, \ldots, k$, thus $M=M_{k}$. Proceed by induction on $j$ to show that $E\left(M_{j}\right)$ and $O\left(M_{j}\right)$ are residue equivalent for $M_{j}$. 
Observe that $E\left(\left\{m_{1}\right\}\right)=\{0\}$ and $O\left(\left\{m_{1}\right\}\right)=\left\{m_{1}\right\}$ are residue equivalent for $\left\{m_{1}\right\}$. Let $j \leqslant k-1$ be such that $E\left(M_{j}\right)$ and $O\left(M_{j}\right)$ are residue equivalent for $M_{j}$. Then we have

$$
\begin{aligned}
& E\left(M_{j+1}\right)=E\left(M_{j}\right) \cup\left\{m_{j+1}+o: o \in O\left(M_{j}\right)\right\} \\
& O\left(M_{j+1}\right)=O\left(M_{j}\right) \cup\left\{m_{j+1}+e: e \in E\left(M_{j}\right)\right\} .
\end{aligned}
$$

Observe that $E\left(M_{j}\right)$ and $O\left(M_{j}\right)$ are residue equivalent for $M_{j}$, and $\left\{m_{j+1}+o: o \in O\left(M_{j}\right)\right\}$ and $\left\{m_{j+1}+e: e \in E\left(M_{j}\right)\right\}$ are also residue equivalent for $M_{j}$. Consequently, $E\left(M_{j+1}\right)$ and $O\left(M_{j+1}\right)$ are residue equivalent for $M_{j}$.

Observe that both $E\left(M_{j+1}\right)$ and $O\left(M_{j+1}\right)$ are residue equivalent to $E\left(M_{j}\right) \cup O\left(M_{j}\right)$ for $m_{j+1}$, hence residue equivalent to each other for $m_{j+1}$. This implies that $E\left(M_{j+1}\right)$ and $O\left(M_{j+1}\right)$ are residue equivalent for $M_{j+1}$. By an immediate induction, we get that $E(M)$ and $O(M)$ are residue equivalent for $M$.

Since the multiset $M$ of residues modulo $n$ is fair, we deduce from Theorem 2 that $E(M)$ and $O(M)$ have different counts for the residue 0 modulo $n$. Hence, the multisets $E(M)$ and $O(M)$ are not residue equivalent for $n$. Define $X$ and $Y$ as the sets consisting of the minimal non-negative integers such that $X$ is residue equivalent to $E(M)$ for $M \cup\{n\}$ and $Y$ is residue equivalent to $O(M)$ for $M \cup\{n\}$.

\subsection{Block equivalence}

Notation. For a finite, non-empty set $M$ of positive integers, $\operatorname{lcm}(M)$ denotes the least common multiple of the elements of $M$. We set $\operatorname{lcm}(\varnothing)=1$.

Definition. Let $s$ be a base and let $M$ be a set of positive integers. Let $u$ and $v$ be blocks in $\mathcal{L}\left(\ell, B_{s}\right)$, where $\ell$ is a multiple of $\operatorname{lcm}(M)$. Then, $u$ and $v$ are block equivalent for $M$ if and only if, for each $m \in M$ and for each block $z$ of length $m$ on $B_{s}$, the number of occurrences of $z$ in $(u ; m)$ is the same as the number of occurrences of $z$ in $(v ; m)$. When $M=\{m\}$ is a singleton, we say that $u$ and $v$ are block equivalent for $m$.

Lemma 4. Let $s$ be a base, $M$ be a finite set of positive integers and $n$ be a positive integer that does not divide any element of $M$. There are blocks $u$ and $v$ of digits in base $s$ which are block equivalent for $M$ and not block equivalent for $n$, and their length is a multiple of each of the elements in $M$ and $n$.

Proof. The first possibility is that $n$ is equal to 1 and hence $M$ is empty. Then the two blocks (0) and (1) of length 1 satisfy the conclusions of the lemma.

The second possibility is that $n$ is greater than 1 . As in the proof of Lemma 3, enlarging $M$ if necessary, we may assume that the multiset of residues of $M$ modulo $n$ is fair, and hence not empty. By Lemma 3, let $X$ and $Y$ be sets of non-negative integers that are residue equivalent for $M$ but not for $n$. Use concatenations of $\# X$ many blocks of length $\ell$, where $\ell$ is a multiple of $\operatorname{lcm}(M \cup\{n\})$ and strictly greater than the maximum of $X$ and $Y$. We represent an element $x \in X$ by the block $w_{x}$ consisting of $\ell-1$ many 0 s and a unique 1 at position $x$. Recall that the initial position is numbered by 0 and the last position by $\ell-1$. We concatenate these blocks in the order of the elements of $X$, but any order would do. We define the block for $Y$ similarly.

For each $x$ in $X$ and each $m$ in $M$, the sequence $\left(w_{x} ; m\right)$ is composed of $\ell / m$ many blocks. All but one of these are the identically equal to 0 block. The remaining element of $\left(w_{x} ; m\right)$ contains a 1 at position $r$, where $r=x \bmod m$. Since $X$ and $Y$ are residue equivalent for $M$, the 
blocks representing $X$ and $Y$ are block equivalent for $M$. Similarly, the blocks representing $X$ and $Y$ are not block equivalent for $n$, by the hypothesis that $X$ and $Y$ are not residue equivalent for $n$ and the above argument.

We point out that the two blocks $u$ and $v$ defined in the proof of the Lemma 4 are binary blocks, then a fortiori blocks of digits in any base $s$.

Definition. Let $V$ be a finite set and let $w$ be a block in $\mathcal{L}(\ell, V)$. For $v \in V$, let $\operatorname{occ}(w, v)$ be the number of occurrences in $w$ of $v$. The simple discrepancy of $w$ for the set $V$ is

$$
D(w, V)=\max \left\{\left|\frac{\operatorname{occ}(w, v)}{\ell}-\frac{1}{\# V}\right|: v \in V\right\} .
$$

Definition. A block $w$ of length $\ell$ of digits in a finite set $V$ is balanced for an integer $m$ and $V$ if $\ell$ is a multiple of $m$ and $D((w ; m), \mathcal{L}(m, V))=0$. A block $w$ is balanced for a set $M$ of integers and a finite set $V$ if it is balanced for every integer in $M$ and $V$. A set $W$ of blocks of length $\ell$ is balanced for a set $M$ of integers and a finite set $V$ if $\ell$ is a multiple of each element of $M$ and the concatenation of the blocks in $W$ (in any order) is balanced for $M$ and $V$.

Suppose that $W$ is a subset of $\mathcal{L}\left(\ell, B_{s}\right)$ and $\ell$ is divisible by $m$. Consider the uniform measure $\mu_{W}$ on the infinite sequences of elements of $W$. If $W$ is balanced for $m$ and $B_{s}$, then $\mu_{W}$-almost-every infinite sequence of elements from $W$ is simply normal to base $s^{m}$, when parsed as an infinite sequence of elements in $\mathcal{L}\left(m, B_{s}\right)$,

Lemma 5. Let s be a base, $M$ be a finite set of positive integers and $n$ be a positive integer that does not divide any element of $M$. There is a set $U=U(s, M, n)$ and a positive integer $\ell_{U}$, which may be chosen arbitrarily large, such that

- $U$ is balanced for $M$ and not balanced for $n$,

- if $s$ is odd, then $U=\mathcal{L}\left(\ell_{U}, B_{s}\right) \backslash\{z\}$ with $z$ even,

- if $s$ is even, then $U=\mathcal{L}\left(\ell_{U}, B_{s}\right) \backslash\{z, \tilde{z}\}$ with $z$ even, $\tilde{z}$ odd and $z<\tilde{z}$.

Furthermore, $\ell_{U}$ and $U$ are uniformly computable from of $s, M$ and $n$.

The fact that $U$ and $\ell_{U}$ may be chosen arbitrarily large is crucial in Section 4 .

Proof. If $s$ is equal to 2 and $n$ is equal to 1 then let $u$ and $v$ be the $n$-inequivalent blocks $(0,1)$ and $(1,1)$. Otherwise, let $u$ and $v$ be blocks of digits in base $s$ ensured by Lemma 4 to be block equivalent for $M$ but not for $n$. The length $\ell$ of the blocks $u$ and $v$ is a positive integer that is divisible by all of the elements of $M$ and also by $n$. Fix any positive integer $c$ and let $w_{0}$ be a block of length $2 c \ell s^{\ell}$ obtained by concatenating $2 c$ many instances of each of the $s^{\ell}$ elements of $\mathcal{L}\left(\ell, B_{s}\right)$ in some order. By symmetry, each element of $s$ occurs in $w_{0}$ exactly as often as any other element does. Similarly, if $k$ divides $\ell$, then $\left(w_{0} ; k\right)$ can be obtained by concatenating the elements of $\mathcal{L}\left(\ell / k, B_{s^{k}}\right)$ in the order naturally induced by $w_{0}$ and so each digit in base $s^{k}$ appears in $\left(w_{0} ; k\right)$ exactly as often as any other digit does. Thus, for each $k$ that divides $\ell$, the block $w_{0}$ is balanced for $k$.

Now let $W$ be the set of blocks in $\mathcal{L}\left(2 c \ell s^{\ell}, B_{s}\right)$ obtained by concatenation of $2 c$ many instances of $u$ and $2 c$ many instances of each of the elements in $\mathcal{L}\left(\ell, B_{s}\right) \backslash\{v\}$. Observe that each element $w$ in $W$ consists of $4 c$ instances of $u$ and $2 c$ instances of each of the blocks in 
$\mathcal{L}\left(\ell, B_{s}\right) \backslash\{u, v\}$. No instances of $v$ have been used. Since $u$ and $v$ are block equivalent for $M$, $w_{0}$ is block equivalent to every $w \in W$ for $M$. Since $w_{0}$ is balanced for $M$, each $w \in W$ is also balanced for $M$. Similarly, since $u$ and $v$ are not block equivalent for $n$ and $w_{0}$ is balanced for $n$, each $w \in W$ is not balanced for $n$. In fact, all the elements in $W$ are identically imbalanced for $n$. Then, there is a block $t \in \mathcal{L}\left(n, B_{s}\right)$ and a positive rational constant $\gamma$ such that for any two blocks $w$ and $\tilde{w}$ in $W$,

$$
\frac{\operatorname{occ}((w ; n), t)}{|w| / n}=\frac{\operatorname{occ}((\tilde{w} ; n), t)}{|\tilde{w}| / n}<\frac{1}{s^{n}}-\gamma
$$

Let $z$ and $\tilde{z}$ be the lexicographically least pair of blocks in $W$ such that $z$ ends with an even digit, $\tilde{z}$ ends with an odd digit and $z$ is less than $\tilde{z}$. The existence of these blocks $z$ and $\tilde{z}$ follows from the fact that the blocks $u$ and $v$ have length $\ell$ greater than or equal to 2 . This is ensured by the choice of $u$ and $v$ in the special case of $s=2$ and $n=1$, and by Lemma 4 in all the other cases.

If $s$ is even, then $z$ is even and $\tilde{z}$ is odd. If $s$ is odd, then $z$ is even, since the sum of its digits is even (we have concatenated an even number of instances of each block). Let the length $\ell_{U}$ be equal to $2 c \ell s^{\ell}$ (which is the length of $z$ and $\tilde{z}$ ). If $s$ is odd, let $U=\mathcal{L}\left(\ell_{U}, B_{s}\right) \backslash\{z\}$. If $s$ is even, let $U=\mathcal{L}\left(\ell_{U}, B_{s}\right) \backslash\{z, \tilde{z}\}$.

We argue for the case $s$ is even. Since $U \cup\{z, \tilde{z}\}$ is balanced for $M$ and both $z$ and $\tilde{z}$ are also balanced for $M$, we deduce that $U$ is also balanced for $M$. Similarly, $U \cup\{z, \tilde{z}\}$ is balanced for $n$ and $z$ and $\tilde{z}$ are identically imbalanced for $n$, thus $U$ is not balanced for $n$. The case $s$ is odd is similar.

Finally, the computability of $U$ follows from the fact that $z$ and $\tilde{z}$ are uniformly computable in terms of $s, M$ and $n$.

\subsection{A lower bound for simple discrepancy in Cantor sets}

Our next lemma is a classical statement saying that, for a finite set $V$, if $\ell$ is large enough then a large proportion of blocks of length $\ell$ of digits from the set $V$ have small simple discrepancy.

Lemma 6 (see Theorem 148, [8]). For any finite set $V$, for any positive real numbers $\epsilon$ and $\delta$, there is a positive integer $\ell_{0}$ such that for all $\ell \geqslant \ell_{0}$,

$$
\#\{v \in \mathcal{L}(\ell, V): D(v, V)<\epsilon\}>(1-\delta)(\# V)^{\ell} .
$$

Furthermore, $\ell_{0}$ is a computable function of $V, \epsilon$ and $\delta$.

Our second lemma will be used to ensure simple normality with respect to bases $s^{m}$, with $m$ in a finite set $M$.

Lemma 7. Let $s$ be a base, $M$ be a finite set of positive integers and $n$ be a positive integer that does not divide any element of $M$. Let $U$ be as in Lemma 5 and let $\ell_{U}$ be the length of the elements of $U$.

For any positive real numbers $\epsilon$ and $\delta$, there is a positive integer $\ell_{0}$ such that for all $\ell \geqslant \ell_{0}$,

$$
\#\left\{u \in \mathcal{L}\left(\ell \ell_{U}, B_{s}\right):\left(u ; \ell_{U}\right) \in \mathcal{L}(\ell, U) \text { and } \forall m \in M, D\left((u ; m), \mathcal{L}\left(m, B_{s}\right)\right)<\epsilon\right\}>(1-\delta)(\# U)^{\ell} .
$$

Furthermore, $\ell_{0}$ is a computable function of $s, M, n, \epsilon$ and $\delta$. 
Keeping its notation, Lemma 7 asserts that, if $\ell$ is large enough, then, an arbitrarily large proportion of the $(\# U)^{\ell}$ blocks of length $\ell$ of elements of the set $U$ has, for each $m \in M$, the property that, viewed as blocks of length $\ell \ell_{U} / m$ of digits in $\left\{0,1, \ldots, s^{m}-1\right\}$, they have arbitrarily small simple discrepancy for the base $s^{m}$. This holds because both, $z$ and $\tilde{z}$, are balanced for $m$.

Proof. Consider a block $u \in \mathcal{L}\left(\ell \ell_{U}, B_{s}\right)$ such that $\left(u ; \ell_{U}\right) \in \mathcal{L}(\ell, U)$. Let $m \in M$ and $d \in \mathcal{L}\left(m, B_{s}\right)$. We count the number of occurrences in $(u ; m)$ of $d$,

$$
\operatorname{occ}((u ; m), d)=\sum_{w \in U} \operatorname{occ}\left(\left(u ; \ell_{U}\right), w\right) \operatorname{occ}((w ; m), d) .
$$

If $D\left(\left(u ; \ell_{U}\right), U\right)<\epsilon_{1}$ then, by the definition of discrepancy $D$, for all $w \in U$,

$$
\frac{\operatorname{occ}\left(\left(u ; \ell_{U}\right), w\right)}{\ell}<\frac{1}{\# U}+\epsilon_{1} .
$$

Then,

$$
\operatorname{occ}((u ; m), d)<\ell\left(\frac{1}{\# U}+\epsilon_{1}\right) \sum_{w \in U} \operatorname{occ}((w ; m), d) .
$$

Since $U$ is balanced for $M$,

$$
\sum_{w \in U} \operatorname{occ}((w ; m), d)=\frac{\# U\left(\ell_{U} / m\right)}{\# \mathcal{L}\left(m, B_{s}\right)}=\frac{\# U\left(\ell_{U} / m\right)}{s^{m}} .
$$

Then,

$$
\begin{aligned}
\operatorname{occ}((u ; m), d) & <\ell\left(\frac{1}{\# U}+\epsilon_{1}\right) \frac{\# U\left(\ell_{U} / m\right)}{s^{m}} \\
& <\ell\left(\frac{1}{s^{m}}+\frac{\epsilon_{1} \# U}{s^{m}}\right) \frac{\ell_{U}}{m} .
\end{aligned}
$$

Since $\ell \ell_{U} / m$ is the length of $(u ; m)$, we deduce that

$$
\frac{\operatorname{occ}((u ; m), d)}{|(u ; m)|}<\frac{1}{s^{m}}+\epsilon_{1} s^{\ell_{U}} .
$$

We obtain the analogous lower bound on $\frac{\operatorname{occ}((u ; m), d)}{|(u ; m)|}$ similarly. Thus, for any $\epsilon>0$ and any $u \in \mathcal{L}\left(\ell \ell_{U}, B_{s}\right)$ satisfying $D\left(\left(u ; \ell_{U}\right), U\right)<\epsilon s^{-\ell_{U}}$, we have $D\left((u ; m), B_{s}\right)<\epsilon$.

Now, let $\epsilon$ and $\delta$ be positive real numbers. By Lemma 6, there is an $\ell_{0}$ such that for all $\ell \geqslant \ell_{0}$

$$
\#\left\{u \in \mathcal{L}(\ell, U): D(u, U)<\epsilon s^{-\ell_{U}}\right\}>(1-\delta)(\# U)^{\ell} .
$$

Equivalently,

$$
\#\left\{u \in \mathcal{L}\left(\ell \ell_{U}, B_{s}\right):\left(u, \ell_{U}\right) \in \mathcal{L}(\ell, U) \text { and } D\left(\left(u ; \ell_{U}\right), U\right)<\epsilon s^{-\ell_{U}}\right\}>(1-\delta)(\# U)^{\ell} .
$$

Hence,

$$
\#\left\{u \in \mathcal{L}\left(\ell \ell_{U}, B_{s}\right):\left(u, \ell_{U}\right) \in \mathcal{L}(\ell, U) \text { and } D\left((u ; m), \mathcal{L}\left(m, B_{s}\right)\right)<\epsilon\right\}>(1-\delta)(\# U)^{\ell},
$$

as required. 
Our third lemma is the key ingredient to deny simple normality to the base $s^{n}$.

Lemma 8. Let $s$ be a base, $M$ be a finite set of positive integers and $n$ be a positive integer that does not divide any element of $M$. Let $U$ be fixed as in Lemma 5 and let $\ell_{U}$ be the length of the elements of $U$.

There is a positive real number $\epsilon$ and an element $d$ in $\mathcal{L}\left(n, B_{s}\right)$ such that for any positive real number $\delta$, there is a positive integer $\ell_{0}$ such that for all $\ell \geqslant \ell_{0}$,

$$
\#\left\{u \in \mathcal{L}\left(\ell \ell_{U}, B_{s}\right):\left(u ; \ell_{U}\right) \in \mathcal{L}(\ell, U) \text { and } \frac{o c c((u ; n), d)}{|(u ; n)|}<\frac{1}{s^{n}}-\epsilon\right\}>(1-\delta)(\# U)^{\ell},
$$

Furthermore, $\epsilon, d$ and $\ell_{0}$ are computable functions of $s, M$ and $n$.

We point out that $\epsilon$ in Lemma 8 does not depend on $\delta$.

Proof. We argue as in Lemma 7. Consider a block $u \in \mathcal{L}\left(\ell \ell_{U}, B_{s}\right)$ and a positive real number $\epsilon_{1}$ such that $\left(u ; \ell_{U}\right) \in \mathcal{L}(\ell, U)$ and $D\left(\left(u ; \ell_{U}\right), U\right)<\epsilon_{1}$. As above, for any $d \in \mathcal{L}\left(n, B_{s}\right)$, we have

$$
\operatorname{occ}((u ; n), d)<\ell\left(\frac{1}{\# U}+\epsilon_{1}\right) \sum_{w \in U} \operatorname{occ}((w ; n), d) .
$$

Since $U$ is not balanced for $n$, there is some $d \in \mathcal{L}\left(n, B_{s}\right)$ and a positive constant $c$ such that

$$
\sum_{w \in U} \operatorname{occ}((w ; n), d)=\frac{\# U\left(\ell_{U} / n\right)}{\# \mathcal{L}\left(n, B_{s}\right)}-c=\frac{\# U\left(\ell_{U} / n\right)}{s^{n}}-c .
$$

Thus,

$$
\begin{aligned}
& \operatorname{occ}((u ; n), d)<\ell\left(\frac{1}{\# U}+\epsilon_{1}\right)\left(\frac{\# U\left(\ell_{U} / n\right)}{s^{n}}-c\right) \text { and } \\
& \frac{\operatorname{occ}((u ; n), d)}{\ell \ell_{U} / n}<\frac{1}{s^{n}}-\frac{c}{\# U \ell_{U} / n}+\epsilon_{1} s^{\ell_{U}} .
\end{aligned}
$$

If $\epsilon_{1}$ is sufficiently small, then

$$
\frac{\operatorname{occ}((u ; n), d)}{\ell \ell_{U} / n}<\frac{1}{s^{n}}-\frac{c}{2 \# U \ell_{U} / n} .
$$

Let $\epsilon$ equal $\frac{c}{2 \# U \ell_{U} / n}$. The proof is completed by application of Lemma 6 ,

\subsection{Exponential sums on Cantor sets}

Notation. We let $e(x)$ denote $\mathrm{e}^{2 \pi i x}$. We use $\langle b ; r\rangle$ to denote $\lceil b / \log r\rceil$, where $\log$ refers to logarithm in base e. We say that a rational number $x$ in the unit interval is $s$-adic if $x=\sum_{j=1}^{a} d_{j} s^{-j}$ for digits $d_{j}$ in $\{0, \ldots, s-1\}$. In this case, we say that $x$ has precision $a$.

Lemma 9 (Hilfssatz 5, [15]). Let $r$ and $s$ be multiplicatively independent bases. There is a constant $c$, with $0<c<1 / 2$, depending only on $r$ and $s$, such that for all positive integers $k$ and $l$ with $l \geqslant s^{k}$ and for every positive integer $n$,

$$
\sum_{p=0}^{n-1} \prod_{q=k+1}^{\infty}\left|\cos \left(\pi r^{p} l / s^{q}\right)\right| \leqslant 2 n^{1-c} .
$$


Furthermore, $c$ is a computable function of $r$ and $s$ 团

Lemma 10 is our analytic tool to control discrepancy for multiplicatively independent bases. It originates in Schmidt's work [15]. Our proof adapts the version given by Pollington [13].

Definition. For integers $a, \ell$, sets $R, T$ and a real number $x$, set

$$
A(x, R, T, a, \ell)=\sum_{t \in T} \sum_{r \in R}\left|\sum_{j=\langle a ; r\rangle+1}^{\langle a+\ell ; r\rangle} e\left(r^{j} t x\right)\right|^{2}
$$

Lemma 10. Let $s$ be a base greater than 2. If $s$ is odd, then let $U$ be $B_{s} \backslash\{z\}$ for some $z$ in $B_{s}$ such that $z$ even. Else, if $s$ is even, then let $U$ be $B_{s} \backslash\{z, \tilde{z}\}$ for some $z$ and $\tilde{z}$ in $B_{s}$ such that $z$ is even and $\tilde{z}$ is odd. Let $R$ be a finite set of bases multiplicatively independent to $s$, $T$ be a finite set of non-zero integers and a be a non-negative integer. Let $x$ be s-adic with precision $\langle a ; s\rangle$.

For every positive real number $\delta$ there is a length $\ell_{0}$ such that for all $\ell \geqslant \ell_{0}$, there are at least $(1-\delta)(\# U)^{k}$ blocks $v$ in $\mathcal{L}(k, U)$ for $k=\langle a+\ell ; s\rangle-\langle a ; s\rangle$ such that $A\left(x_{v}, R, T, a, \ell\right) \leqslant \ell^{2-c(R, s) / 4}$, for $x_{v}=x+s^{-(\langle a ; s\rangle+1)} \sum_{j=0}^{k-1} v_{j} s^{-j}$ and $c(R, s)$ the minimum of the constants $c$ in Lemma 9 for pairs $r, s$ with $r \in R$.

Furthermore, $\ell_{0}$ is a computable function of $s, U, R$ and $T$ and thereby does not depend on a nor on $x$.

Proof. We abbreviate $A(x, R, T, a, \ell)$ by $A(x)$, abbreviate $(a+\ell)$ by $b$ and $\mathcal{L}(\langle b ; s\rangle-\langle a ; s\rangle, U)$ by $\mathcal{L}$. To provide the needed $\ell_{0}$ we estimate the mean value of $A(x)$ on the set of numbers $x_{v}$. We need an upper bound for

$$
\sum_{v \in \mathcal{L}} A\left(x_{v}\right)=\sum_{v \in \mathcal{L}} \sum_{t \in T} \sum_{r \in R}\left|\sum_{j=\langle a ; r\rangle+1}^{\langle b ; r\rangle} e\left(r^{j} t x_{v}\right)\right|^{2}=\sum_{v \in \mathcal{L}} \sum_{t \in T} \sum_{r \in R} \sum_{g=\langle a ; r\rangle+1}^{\langle b ; r\rangle} \sum_{j=\langle a ; r\rangle+1}^{\langle b ; r\rangle} e\left(\left(r^{j}-r^{g}\right) t x_{v}\right) .
$$

Our main tool is Lemma 9, but it does not apply to all the terms $A\left(x_{v}\right)$ in the sum. We will split $\sum_{v \in \mathcal{L}} A\left(x_{v}\right)$ into two smaller sums $\sum_{v \in \mathcal{L}} B\left(x_{v}\right)$ and $\sum_{v \in \mathcal{L}} C\left(x_{v}\right)$, so that a straightforward analysis applies to the first, and Lemma 9 applies to the other. Let $p$ be the least integer satisfying $r^{p-1} \geqslant 2|t|$ for every $t \in T$ and $r^{p} \geqslant s^{2}+1$ for every $r \in R$.

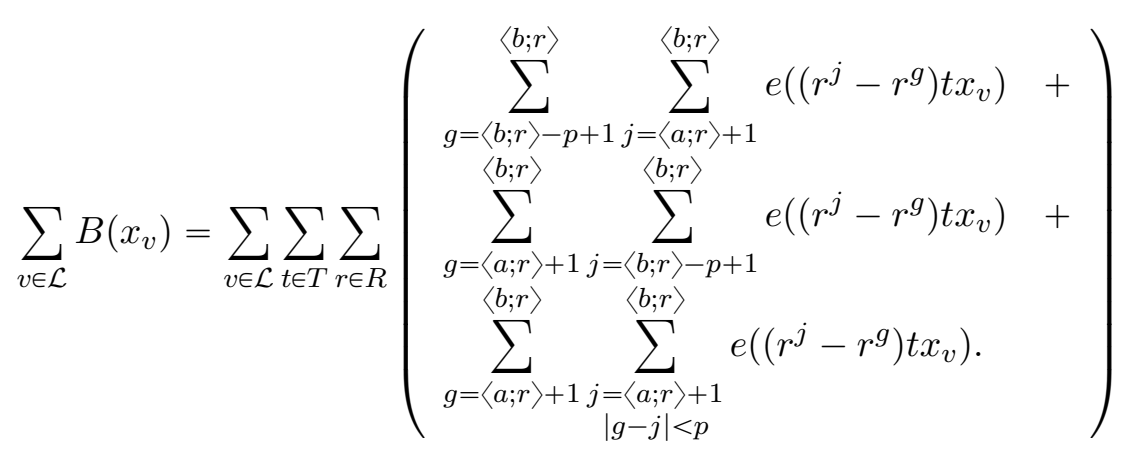

\footnotetext{
${ }^{\ddagger}$ Actually, Schmidt asserts the computability of $c$ in separate paragraph (page 309 in the same article): "Wir stellen zunächst fest, daß man mit etwas mehr Mühe Konstanten $a_{20}(r, s)$ aus Hilfssatz 5 explizit berechnen könnte, und daß dann $x$ eine eindeutig definierte Zahl ist."
} 
We obtain the following bounds. The first inequality uses that each term in the explicit definition of $B(x)$ has norm less than or equal to 1 . Recall, $b=a+\ell$.

$$
\begin{aligned}
\sum_{v \in \mathcal{L}}|B(x)| & \leqslant \sum_{v \in \mathcal{L}} \sum_{t \in T} \sum_{r \in R} 4 p(\langle b ; r\rangle-\langle a ; r\rangle) \\
& \leqslant \sum_{v \in \mathcal{L}} \# T \# R 8 p \ell \\
& \leqslant \# T \# R 8 p \ell(\# U)^{\langle b ; s\rangle-\langle a ; s\rangle} .
\end{aligned}
$$

The other sum is as follows.

$$
\begin{aligned}
\sum_{v \in \mathcal{L}} C\left(x_{v}\right) & =\sum_{v \in \mathcal{L}} \sum_{t \in T} \sum_{r \in R} \sum_{g=\langle a ; r\rangle+1}^{\langle b ; r\rangle-p} \sum_{\substack{j=\langle a ; r\rangle+1 \\
|j-g| \geqslant p}}^{\langle b ; r\rangle-p} e\left(\left(r^{j}-r^{g}\right) t x_{v}\right) \\
& =\sum_{t \in T} \sum_{r \in R} \sum_{g=\langle a ; r\rangle+1}^{\langle b ; r\rangle-p} \sum_{\substack{\langle b ; r\rangle-p \\
j=\langle a ; r\rangle+1 \\
|j-g| \geqslant p}}^{\langle b \in \mathcal{L}} e\left(\left(r^{j}-r^{g}\right) t x_{v}\right) .
\end{aligned}
$$

For fixed $j$ and $g$, we have the following identity:

$$
\sum_{v \in \mathcal{L}} e\left(\left(r^{j}-r^{g}\right) t x_{v}\right)=e\left(\left(r^{j}-r^{g}\right) t x\right) \prod_{k=\langle a ; s\rangle+1}^{\langle b ; s\rangle} \sum_{d \in U} e\left(\frac{d t\left(r^{j}-r^{g}\right)}{s^{k}}\right) .
$$

Since $\left|\sum_{x \in X} e(x)\right|=\left|\sum_{x \in X} e(-x)\right|$ holds for any finite set $X$ of real numbers, we can bound the sums over $g$ and $j$ as follows:

$$
\begin{aligned}
\left|\sum_{v \in \mathcal{L}} C\left(x_{v}\right)\right| & \leqslant \sum_{t \in T} \sum_{r \in R} \sum_{j=\langle a ; r\rangle+1}^{\langle b ; r\rangle-p} \sum_{\substack{g=\langle a ; r\rangle+1 \\
|j-g| \geqslant p}}^{\langle b ; r\rangle-p} \prod_{k=\langle a ; s\rangle+1}^{\langle b ; s\rangle}\left|\sum_{d \in U} e\left(\frac{d t\left(r^{j}-r^{g}\right)}{s^{k}}\right)\right| \\
& \leqslant 2 \sum_{t \in T} \sum_{r \in R} \sum_{j=p}^{\langle b ; r\rangle-\langle a ; r\rangle-p} \sum_{g=1}^{\langle b ; r\rangle-\langle a ; r\rangle-p-j} \prod_{k=\langle a ; s\rangle+1}^{\langle b ; s\rangle}\left|\sum_{d \in U} e\left(\frac{d t r^{\langle a ; r\rangle} r^{g}\left(r^{j}-1\right)}{s^{k}}\right)\right| .
\end{aligned}
$$

Now, we use the properties of $U$ to show that $\left|\sum_{d \in U} e(d x)\right| \leqslant \frac{1}{2} \# U|1+e(x)|$. We will also show that $\# U$ is even and thus $\frac{1}{2} \# U$ is a positive integer. We consider the odd and even cases for $s$ separately.

Suppose that $s$ is odd. By hypothesis $U$ is $\{0, \ldots, z-1, z+1, \ldots, s-1\}$ and $z$ is even. Hence, $\# U=s-1$ and is even. We parse our sum in pairs:

$$
\begin{aligned}
\sum_{d \in U} e(d x) & =\sum_{\substack{d \in U \\
d<z}} e(d x)+\sum_{\substack{d \in U \\
z<d}} e(d x) \\
& =\sum_{\substack{d \in U \\
d<z \\
d \text { even }}} e(d x)(1+e(x))+\sum_{\substack{d \in U \\
z<d \\
d \text { odd }}} e(d x)(1+e(x)) .
\end{aligned}
$$

We conclude that $\left|\sum_{d \in U} e(d x)\right| \leqslant \frac{1}{2} \# U|1+e(x)|$. 
Suppose that $s$ is even. Then, $z<\tilde{z}$ and $U$ is $\{0, \ldots, z-1, z+1, \ldots, \tilde{z}-1, \tilde{z}+1, \ldots, s-1\}$, where $z$ is even and $\tilde{z}$ is odd. Hence, $\# U=s-2$ and is even. Again, we parse our sum in pairs:

$$
\begin{aligned}
\sum_{d \in U} e(d x) & =\sum_{\substack{d \in U \\
d<z}} e(d x)+\sum_{\substack{d \in U \\
z<d<\tilde{z}}} e(d x)+\sum_{\substack{d \in U \\
\tilde{z}<d}} e(d x) \\
& =\sum_{\substack{d \in U \\
d<z \\
d \text { even }}} e(d x)(1+e(x))+\sum_{\substack{d \in U \\
z<d<\tilde{z} \\
d \text { odd }}} e(d x)(1+e(x))+\sum_{\substack{d \in U \\
\tilde{z}<d \\
d \text { even }}} e(d x)(1+e(x)) .
\end{aligned}
$$

Again, we conclude that $\left|\sum_{d \in U} e(d x)\right| \leqslant \frac{1}{2} \# U|1+e(x)|$.

To simplify the expressions, let $L$ denote $\left(r^{j}-1\right) r^{\langle a ; r\rangle} t$. Then,

$$
\begin{aligned}
\left|\sum_{v \in \mathcal{L}} C\left(x_{v}\right)\right| & \leqslant 2 \sum_{t \in T} \sum_{r \in R} \sum_{j=p}^{\langle b ; r\rangle-\langle a ; r\rangle-p} \sum_{g=1}^{\langle b ; r\rangle-\langle a ; r\rangle-p-j} \prod_{k=\langle a ; s\rangle+1}^{\langle b ; s\rangle}\left|\sum_{d \in U} e\left(d L r^{g} s^{-k}\right)\right| \\
& \leqslant 2 \sum_{t \in T} \sum_{r \in R} \sum_{j=p}^{\langle b ; r\rangle-\langle a ; r\rangle-p} \sum_{g=1}^{\langle b ; r\rangle-\langle a ; r\rangle-p-j} \prod_{k=\langle a ; s\rangle+1}^{\langle b ; s\rangle} \frac{\# U}{2}\left|1+e\left(L r^{g} s^{-k}\right)\right| .
\end{aligned}
$$

By the double angle identities,

$$
\left|\sum_{v \in \mathcal{L}} C\left(x_{v}\right)\right| \leqslant 2(\# U)^{\langle b ; s\rangle-\langle a ; s\rangle} \sum_{t \in T} \sum_{r \in R} \sum_{j=p}^{\langle b ; r\rangle-\langle a ; r\rangle-p} \sum_{g=1}^{\langle b ; r\rangle-\langle a ; r\rangle-p-j} \prod_{k=\langle a ; s\rangle+1}^{\langle b ; s\rangle}\left|\cos \left(\pi L r^{g} s^{-k}\right)\right| .
$$

The following upper bound on the value of $L$ for $r, j$ and $t$ is ensured by the choice of $p$. Let $T_{\max }$ be the maximum of the absolute values of the elements of $T$.

$$
\begin{aligned}
L r^{g} s^{-\langle b ; s\rangle} & \leqslant\left(r^{j}-1\right) r^{\langle a ; r\rangle} t^{g} s^{-\langle b ; s\rangle} \\
& \leqslant r^{j} r^{\langle a ; r\rangle} t r^{\langle b ; r\rangle-\langle a ; r\rangle-p-j} s^{-\langle b ; s\rangle}=t r^{\langle b ; r\rangle-p} s^{-\langle b ; s\rangle} \\
& \leqslant T_{\max } r^{[b / \log r]} s^{-[b / \log s]} r^{-p} \\
& \leqslant T_{\max } r^{1-p} \\
& \leqslant 1 / 2 \quad(\text { an ensured condition on } p) .
\end{aligned}
$$

Using this upper bound, for every $r, j$ and $t$ above, $L r^{g} s^{-(\langle b ; s\rangle+k)} \leqslant 2^{-(k+1)}$. We conclude that

$$
\prod_{k=\langle b ; s\rangle+1}^{\infty}\left|\cos \left(\pi L r^{g} s^{-k}\right)\right| \geqslant \prod_{k=1}^{\infty}\left|\cos \left(\pi 2^{-(k+1)}\right)\right|
$$

where the right hand side is a positive constant. Then, for all $r, j$ and $t$

$$
\prod_{k=\langle a ; s\rangle+1}^{\langle b ; s\rangle}\left|\cos \left(\pi L r^{g} s^{-k}\right)\right|=\prod_{k=\langle a ; s\rangle+1}^{\infty}\left|\cos \left(\pi L r^{g} s^{-k}\right)\right|\left(\prod_{k=\langle b ; s\rangle+1}^{\infty}\left|\cos \left(\pi L r^{g} s^{-k}\right)\right|\right)^{-1}
$$


which, for an appropriate constant $\tilde{c}$, is at most $\tilde{c} \prod_{k=\langle a ; s\rangle+1}^{\infty}\left|\cos \left(\pi L r^{g} s^{-k}\right)\right|$.

Now, for $r, j$ and $t$, we give a lower bound on the absolute value of $L$.

$$
\begin{aligned}
|L| & \geqslant\left(r^{p}-1\right) r^{\langle a ; r\rangle}=\left(r^{p}-1\right) r^{[a / \log r]} \\
& \geqslant\left(r^{p}-1\right) s^{a / \log s} \\
& \geqslant s^{2+a / \log s} \quad(\text { an ensured condition on } p) \\
& \geqslant s^{\langle a ; s\rangle+1} .
\end{aligned}
$$

Using this lower bound, we can apply Lemma 9

$$
\begin{aligned}
\sum_{g=1}^{\langle b ; r\rangle-\langle a ; r\rangle-p-j} \prod_{k=\langle a ; s\rangle+1}^{\langle b ; s\rangle}\left|\sum_{d \in U} e\left(d L r^{g} s^{-k}\right)\right| & \leqslant \sum_{g=1}^{\langle b ; r\rangle-\langle a ; r\rangle-p-j} \tilde{c} \prod_{k=\langle a ; s\rangle+1}^{\infty}\left|\cos \left(\pi L r^{g} s^{-k}\right)\right| \\
& \leqslant 2 \tilde{c}(\langle b ; r\rangle-\langle a ; r\rangle)^{1-c(R, s) .} .
\end{aligned}
$$

Then,

$$
\begin{aligned}
\left|\sum_{v \in \mathcal{L}} C\left(x_{v}\right)\right| & \leqslant 2(\# U)^{\langle b ; s\rangle-\langle a ; s\rangle} \sum_{t \in T} \sum_{r \in R} \sum_{j=p}^{\langle b ; r\rangle-\langle a ; r\rangle-p} 2 \tilde{c}(\langle b ; r\rangle-\langle a ; r\rangle)^{1-c(R, s)} \\
& \leqslant 16 \tilde{c} \# T \# R \ell^{2-c(R, s)}(\# U)^{\langle b ; s\rangle-\langle a ; s\rangle .}
\end{aligned}
$$

Combining this with the estimate for $\left|\sum_{v \in \mathcal{L}} B\left(x_{v}\right)\right|$ and using that $c(R, s)$ is less than 1 , we have

$$
\sum_{v \in \mathcal{L}} A\left(x_{v}\right) \leqslant(8 p+16 \tilde{c}) \# T \# R \ell^{2-c(R, s)}(\# U)^{\langle b ; s\rangle-\langle a ; s\rangle} .
$$

Therefore, the number of $v \in \mathcal{L}$ such that $A\left(x_{v}\right)>(8 p+16 \tilde{c}) \# T \# R \ell^{2-c(R, s) / 2}$ is at most equal to $\ell^{-c(R, s) / 2}(\# U)^{\langle b ; s\rangle-\langle a ; s\rangle}$. If $\ell$ is greater than $\delta^{-2 / c(R, s)}$ then $\ell^{-c(R, s) / 2}<\delta$. In this case, there are at least $(1-\delta)(\# U)^{(\langle b ; s\rangle-\langle a ; s\rangle)} v \in \mathcal{L}$ for which

$$
A\left(x_{v}\right) \leqslant(8 p+16 \tilde{c}) \# T \# R \ell^{2-c(R, s) / 2} .
$$

If $\ell$ is also greater than $((8 p+16 \tilde{c}) \# T \# R)^{4 / c(R, s)}$, then there are at least $(1-\delta)(\# U)^{(\langle b ; s\rangle-\langle a ; s\rangle)}$ $v \in \mathcal{L}$ for which

$$
A\left(x_{v}\right) \leqslant \ell^{2-c(R, s) / 4} .
$$

This proves the lemma for $\ell_{0}$ equal to the least integer greater than $\delta^{-2 / c(R, s)}$ and greater than $((8 p+16 \tilde{c}) \# T \# R)^{4 / c(R, s)}$.

\subsection{An upper bound for simple discrepancy in Cantor sets}

We apply LeVeque's Inequality, which we state for the special case of simple discrepancy of the digits in the base $s$ expansion of a real number. 
Lemma 11 (LeVeque's inequality, see Theorem 2.4 on page 111 in [10]). Let $s$ be a base, $\ell$ be a positive integer, $w$ be a block in $\mathcal{L}\left(\ell, B_{s}\right)$ and $x$ be a s-adic rational number with precision $a$. Then, letting $x_{w}=x+s^{-a+1} \sum_{j=0}^{\ell-1} w_{j} s^{-j}$,

$$
D\left(w, B_{s}\right) \leqslant\left(\frac{6}{\pi^{2}} \sum_{t=1}^{\infty} \frac{1}{t^{2}}\left|\frac{1}{\ell} \sum_{j=a+1}^{a+\ell} e\left(t s^{j} x_{w}\right)\right|^{2}\right)^{\frac{1}{3}} .
$$

Lemma 12. Let $s$ be a base, $\epsilon$ be a positive real number and $x$ be a s-adic rational number with precision $a$. There is a finite set $T$ of positive integers and a positive real number $\gamma$ such that, for every positive integer $\ell$ and every block $w$ in $\mathcal{L}\left(\ell, B_{s}\right)$, letting $x_{w}=x+s^{-a+1} \sum_{j=0}^{\ell-1} w_{j} s^{-j}$,

$$
\text { if for all } t \in T, \frac{1}{\ell^{2}}\left|\sum_{j=a+1}^{a+\ell} e\left(t s^{j} x_{w}\right)\right|^{2}<\gamma \text { then } D\left(w, B_{s}\right)<\epsilon,
$$

Proof. Since

$$
\left|\frac{1}{\ell} \sum_{j=a+1}^{a+\ell} e\left(t s^{j} x\right)\right|^{2} \leqslant 1
$$

we get, for each integer $k$,

$$
\sum_{t=k+1}^{\infty} \frac{1}{t^{2}}\left|\frac{1}{\ell} \sum_{j=a+1}^{a+\ell} e\left(t s^{j} x\right)\right|^{2} \leqslant \sum_{t=k+1}^{\infty} \frac{1}{t^{2}} \leqslant \int_{k+1}^{\infty} x^{-2} \mathrm{~d} x \leqslant \frac{1}{k+1} .
$$

Set $k=\left\lceil 12 /\left(\epsilon^{3} \pi^{2}\right)\right\rceil$. Assume that

$$
\frac{1}{\ell^{2}}\left|\sum_{j=a+1}^{a+\ell} e\left(t s^{j} x\right)\right|^{2}<\frac{\epsilon^{3}}{2}
$$

for all positive integers $t$ less than or equal to $k$. Then,

$$
\sum_{t=1}^{k} \frac{1}{t^{2}}\left|\frac{1}{\ell} \sum_{j=0}^{\ell-1} e\left(t s^{j} x\right)\right|^{2}+\sum_{t=k+1}^{\infty} \frac{1}{t^{2}}\left|\frac{1}{\ell} \sum_{j=0}^{\ell-1} e\left(t s^{j} x\right)\right|^{2} \leqslant \sum_{t=1}^{k} \frac{1}{t^{2}} \cdot \frac{\epsilon^{3}}{2}+\frac{1}{k+1} \leqslant \epsilon^{3} \frac{\pi^{2}}{12}+\frac{1}{k+1} .
$$

Our choice of $k$ guarantees that $\left(6 / \pi^{2}\left(\left(\epsilon^{3} \pi^{2} / 12\right)+1 /(k+1)\right)\right)^{\frac{1}{3}}<\epsilon$. It then follows from Lemma 11 that $D\left(w, B_{s}\right)<\epsilon$. This proves the lemma with $T=\{1, \ldots, k\}$ and $\gamma=\epsilon^{3} / 2$.

Lemma 13. Let $s$ be a base greater than 2. If $s$ is odd, then let $U$ be $B_{s} \backslash\{z\}$ for some even $z$. Else, if $s$ is even, then let $U$ be $B_{s} \backslash\{z, \tilde{z}\}$ for some even $z$ and some odd $\tilde{z}$ such that $z<\tilde{z}$. Let $R$ be a finite set of bases multiplicatively independent to $s$. Let $x$ be $s$-adic with precision $\langle a ; s\rangle$.

For all positive real numbers $\epsilon$ and $\delta$ there is a length $\ell_{0}$ such that for all $\ell \geqslant \ell_{0}$, there are at least $(1-\delta)(\# U)^{\langle a+\ell ; s\rangle-\langle a ; s\rangle}$ blocks $v \in \mathcal{L}(\langle a+\ell ; s\rangle-\langle a ; s\rangle, U)$ such that for each $r \in R$, the block $u \in \mathcal{L}\left(\langle a+\ell ; r\rangle-\langle a ; r\rangle, B_{r}\right)$ in the expansion of $x+s^{-(\langle a ; s\rangle+1)} \sum_{j=0}^{\langle a+\ell ; s\rangle-\langle a ; s\rangle-1} v_{j} s^{-j}$ in base $r$ satisfies $D\left(u, B_{r}\right)<\epsilon$.

Furthermore, $\ell_{0}$ is a computable function of $s, U$ and $R$ and thereby does not depend on a nor on $x$. 
Proof. Assume given $s, U, R, x$ and $a$ as in the hypothesis. Fix $\epsilon$ and $\delta$ positive real numbers greater than 0. For each base in $r$ in $R$ consider Lemma 12 with input values the base $r$ and the fixed $\epsilon$. Fix a finite set of positive integers $T_{\epsilon}$ and a positive real number $\gamma_{\epsilon}$ that satisfies the conclusion of Lemma 12 simultaneously for all bases $r$ in $R$ and the fixed $\epsilon$.

Apply Lemma 10 with input values $s, U, R, T_{\epsilon}, x$ and $a$. Then there is an $\ell_{0}$ such that for all $\ell \geqslant \ell_{0}$, there are at least $(1-\delta)(\# U)^{k}$ blocks $v \in \mathcal{L}(k, U)$ such that $A\left(x_{v}, R, T_{\epsilon}, a, \ell\right) \leqslant \ell^{2-c(R, s) / 4}$, where $k=\langle a+\ell ; s\rangle-\langle a ; s\rangle, x_{v}=x+s^{-(\langle a ; s\rangle+1)} \sum_{j=0}^{k-1} v_{j} s^{-j}$ and $c(R, s)$ is the minimum of the constants $c$ in Lemma 9 for pairs $r, s$ with $r \in R$.

Fix $\ell$ be such that $\ell \geqslant \ell_{0}$ and $\ell^{-c(R, s) / 4}<\gamma_{\epsilon}$. By definition,

$$
A\left(x_{v}, R, T_{\epsilon}, a, \ell\right)=\sum_{t \in T_{\epsilon}} \sum_{r \in R}\left|\sum_{j=\langle a ; r\rangle+1}^{\langle a+\ell ; r\rangle} e\left(r^{j} t x_{v}\right)\right|^{2} .
$$

Hence, for each $t \in T_{\epsilon}$ and for each $r \in R$,

$$
\frac{1}{\ell^{2}}\left|\sum_{j=\langle a ; r\rangle}^{\langle a+\ell ; r\rangle} e\left(r^{j} t x_{v}\right)\right|^{2}<\gamma_{\epsilon} .
$$

Then, by Lemma 12, for each $r \in R, D\left(u, B_{r}\right)<\epsilon$, where $u$ is the block of digits from position $\langle a ; r\rangle+1$ to position $\langle a+\ell ; r\rangle$ in the expansion of $x_{v}$ in base $r$.

Lemma 14. Let $\epsilon$ be a positive real number, $s$ and $r$ be bases and $a$ and $b$ be positive integers such that $a<b$. Let $q$ be an $s$-adic rational number with precision $\langle b ; s\rangle$ and $x$ be a real number in the interval $\left[q, q+s^{-\langle b ; s\rangle}\right)$. Let $u$ and $v$ in $\mathcal{L}\left(\langle b ; r\rangle-\langle a ; r\rangle+1, B_{r}\right)$ be, respectively, the blocks in the expansions of $q$ and $x$ in base $r$ between the positions $\langle a ; r\rangle$ and $\langle b ; r\rangle$. Let $p$ be a positive integer and let $\tilde{u}$ in $\mathcal{L}\left(\left\langle b ; r^{p}\right\rangle-\left\langle a ; r^{p}\right\rangle+1, B_{r^{p}}\right)$ be the block in the expansion of $q$ in base $r^{p}$ between the positions $\left\langle a ; r^{p}\right\rangle$ and $\left\langle b ; r^{p}\right\rangle$.

If $D\left(u, B_{r}\right), D\left(\tilde{u}, B_{r^{p}}\right), 2 / r^{p}$ and $3 p /|u|$ are all less than $\epsilon$, then $D\left(v, B_{r}\right)<5 \epsilon$.

Proof. Let $\tilde{v}$ in $\mathcal{L}\left(\left\langle b ; r^{p}\right\rangle-\left\langle a ; r^{p}\right\rangle+1, B_{r^{p}}\right)$ be the block in the expansion of $x$ in base $r^{p}$ between the positions $\left\langle a ; r^{p}\right\rangle$ and $\left\langle b ; r^{p}\right\rangle$. Since $0 \leqslant x-q<s^{-\langle b ; s\rangle}$, then $0 \leqslant x-q \leqslant\left(r^{p}\right)^{-\left\langle b ; r^{p}\right\rangle+1}$. Any difference between $\tilde{u}$ and $\tilde{v}$ other than in the last two digits must come from a block of instances of the digit $r^{p}-1$ in the expansion of $q$ in base $r^{p}$ at positions preceding its last two.

Since $D\left(\tilde{u}, B_{r^{p}}\right)<\epsilon$, at most $\left(1 / r^{p}+\epsilon\right)|\tilde{u}|$ digits in $\tilde{u}$ can be equal to $r^{p}-1$. So, $\tilde{u}$ and $\tilde{v}$ agree on all but the last $\left(1 / r^{p}+\epsilon\right)|\tilde{u}|+2$ digits. But then $u$ and $v$ agree on all but the last $\left(1 / r^{p}+\epsilon\right) p|\tilde{u}|+3 p$ digits. Then, for any $d$ in base $r$, the quantity $|\operatorname{occ}(u, d)-\operatorname{occ}(v, d)|$ is less than or equal to $\left(1 / r^{p}+\epsilon\right) p|\tilde{u}|+3 p$. Thus,

$$
\begin{aligned}
\operatorname{occ}(v, d) /|v| & \leqslant \operatorname{occ}(u, d) /|u|+\left(\left(1 / r^{p}+\epsilon\right) p|\tilde{u}|+3 p\right) /|v| \\
& \leqslant(1 / r+\epsilon)+\left(\left(1 / r^{p}+\epsilon\right) p|\tilde{u}|+3 p\right) /|v| \\
& \leqslant(1 / r+\epsilon)+2\left(1 / r^{p}+\epsilon\right)+3 p /|v| \\
& \leqslant 1 / r+5 \epsilon, \text { provided } 2 / r^{p} \text { and } 3 p /|v| \text { are each less than } \epsilon .
\end{aligned}
$$

The lemma follows.

In the next two lemmas, we denote by $\lambda(I)$ the length of a real interval $I$. 
Lemma 15. For any real interval $I$ and base $s$, there is a s-adic subinterval $I_{s}$ such that $\lambda\left(I_{s}\right) \geqslant \lambda(I) /(2 s)$.

Proof. Let $m$ be least such that $1 / s^{m}<\lambda(I)$. Note that $1 / s^{m} \geqslant \lambda(I) / s$, since $1 / s^{m-1} \geqslant \lambda(I)$. If there is a $s$-adic interval of length $1 / s^{m}$ strictly contained in $I$, then let $I_{s}$ be such an interval, and note that $I_{s}$ has length greater than or equal to $\lambda(I) / s$. Otherwise, there must be a non-negative integer $a$ such that $a / s^{m}$ is in $I$ and neither $(a-1) / s^{m}$ nor $(a+1) / s^{m}$ belongs to $I$. Thus, $2 / s^{m}>\lambda(I)$. However, since $1 / s^{m}<\lambda(I)$ and $s \geqslant 2$ then $2 / s^{m+1}<\lambda(I)$. So, at least one of the two intervals $\left[\frac{s a-1}{s^{m+1}}, \frac{s a}{s^{m+1}}\right)$ or $\left[\frac{s a}{s^{m+1}}, \frac{s a+1}{s^{m+1}}\right)$ must be contained in $I$. Denote by $I_{s}$ one with this property. Then, $\lambda\left(I_{s}\right)$ is $\frac{1}{s^{m+1}}=\frac{1}{2 s} \frac{2}{s^{m}}>\lambda(I) /(2 s)$. In either case, the length of $I_{s}$ is greater than or equal to $\lambda(I) /(2 s)$.

Lemma 16. Let $s$ and $t$ be bases and let $I$ be an s-adic interval of length $s^{-\langle b ; s\rangle}$. For $a=b+\lceil\log s+3 \log t\rceil$, there is an $t$-adic subinterval of $I$ of length $t^{-\langle a ; t\rangle}$.

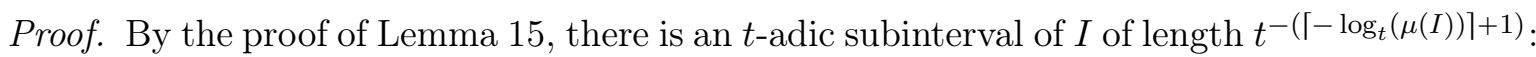

$$
\begin{aligned}
\left\lceil-\log _{t}(\lambda(I))\right\rceil+1 & =\left\lceil-\log _{t}\left(s^{-\langle b ; s\rangle}\right)\right\rceil+1 \\
& =\lceil\langle b ; s\rangle \log s / \log t\rceil+1 \\
& \leqslant\lceil b / \log t+\log s / \log t\rceil+1 \\
& \leqslant\langle b ; t\rangle+\lceil\log s / \log t\rceil+1 .
\end{aligned}
$$

Thus, there is an $t$-adic subinterval of $I$ of length $t^{-(\langle b ; t\rangle+[\log s / \log t\rceil+1)}$. Consider $a=b+\lceil\log s+3 \log t\rceil$,

$$
\begin{aligned}
\langle a ; t\rangle & =\lceil a / \log t\rceil \\
& =\lceil b+\lceil\log s+3 \log t\rceil / \log t\rceil \\
& \geqslant b / \log t+(\log s+3 \log t) / \log t \\
& \geqslant\langle b ; t\rangle+\lceil\log s / \log t\rceil+1 .
\end{aligned}
$$

This inequality is sufficient to prove the lemma.

The next remark follows from direct substitution in Lemma 16 above.

Remark 17. Let $r, s$ and $t$ be bases. Let $b$ be a positive integer and let $a=b+\lceil\log s+3 \log t\rceil$. Then,

$$
\langle a ; r\rangle-\langle b ; r\rangle \leqslant\lceil\log s+3 \log t\rceil / \log r+1 \leqslant 2[\log s+3 \log t\rceil .
$$

\subsection{Simple discrepancy and concatenation}

We record the next three observations without proof.

Lemma 18. Let $\epsilon$ be a positive real, $r$ be a base, $\ell$ a positive integer and $w \in \mathcal{L}\left(\ell, B_{r}\right)$ such that $D\left(w, B_{r}\right)<\epsilon$. Then, for any positive integer $k$ with $k<\epsilon \ell$ and any $u \in \mathcal{L}\left(k, B_{r}\right)$, we have $D\left(w u, B_{r}\right)<2 \epsilon$. 
Lemma 19. Let $\epsilon$ be a positive real, $r$ be a base, and $\left(w_{j}\right)_{j \geqslant 0}$ be an infinite sequence of elements from $\{0,1, \ldots, r-1\}$. Let $\left(b_{t}\right)_{t \geqslant 0}$ be an increasing sequence of positive integers. Suppose that there is an integer $t_{0}$ such that, for all $t>t_{0}$, we have $b_{t+1}-b_{t} \leqslant \epsilon b_{t}$ and $D\left(\left(w_{b_{t}+1}, \ldots, w_{b_{t+1}}\right), B_{r}\right)<\epsilon$. Then,

$$
\lim _{k \rightarrow \infty} D\left(\left(w_{0}, \ldots, w_{k}\right), B_{r}\right) \leqslant 2 \epsilon .
$$

Lemma 20. Let $\epsilon$ be a positive real, $r$ be a base, $d$ be a digit in base $r$ and $\left(w_{j}\right)_{j \geqslant 0}$ be an infinite sequence of elements from $\{0,1, \ldots, r-1\}$. Let $\left(b_{t}\right)_{t \geqslant 0}$ be an increasing sequence of positive integers. Suppose that there is an integer $t_{0}$ such that, for all $t>t_{0}$, we have

$$
\frac{o c c\left(\left(w_{b_{t}+1}, \ldots, w_{b_{t+1}}\right), d\right)}{b_{t+1}-b_{t}}<\frac{1}{r}-\epsilon .
$$

Then,

$$
\liminf _{t \rightarrow \infty} \frac{\operatorname{occ}\left(\left(w_{0}, \ldots, w_{b_{t}}\right), d\right)}{b_{t}+1}<\frac{1}{r}-\frac{\epsilon}{2} .
$$

\section{Existence}

Let $s \mapsto M(s)$ be given as in the hypothesis of the theorem. If for every $s \in \mathcal{S}$, the set $M(s)$ is infinite, then any absolutely normal number satisfies the conclusion of the theorem. Thus, we assume that there is at least one $s \in \mathcal{S}$ for which $M(s)$ is finite. We construct a sequence of intervals by recursion so that the unique real number $x$ in their intersection has the properties stated in the conclusion of the theorem. We define the following sequences indexed by $j$ :

- Fix sequences $\left(\left(s_{j}, n_{j}\right)\right)_{j \geqslant 0}$ and $\left(r_{j}\right)_{j \geqslant 0}$ as follows. In the sequence $\left(\left(s_{j}, n_{j}\right)\right)_{j \geqslant 0}$, the integer $s_{j}$ is an element of $\mathcal{S}$ such that $M\left(s_{j}\right)$ is finite, $n_{j}$ is a positive integer that does not belong to $M\left(s_{j}\right)$ and every such pair appears infinitely often. The sequence $\left(r_{j}\right)_{j \geqslant 0}$ is the enumeration of all of the numbers $s^{m}$, for $s \in \mathcal{S}$ and $m \in M(s)$, in increasing order, including those for which $M(s)$ is infinite.

- For $j \geqslant 0$, set $s_{j}^{*}=s_{j}^{\ell_{U}}$, where $\ell_{U}$ is as in Lemma 5 for $s_{j}, M\left(s_{j}\right)$ and $n_{j}$.

- For $j \geqslant 0$ and the pair $\left(s_{j}, n_{j}\right)$, let $d_{j}$ and $\epsilon_{j}$ be as guaranteed by the conclusion of Lemma 8 for $s_{j}, M\left(s_{j}\right)$ and $n_{j}$.

- For $j \geqslant 0$, let $p_{j}$ be the least positive integer such that for each $k$ less than or equal to $j$, we have $r_{k}^{p_{j}} \geqslant 2(j+1)$.

The recursion uses two additional functions denoted by $\ell(j)$ and $x(j, a, y)$. Let $\ell(j)$ be the least positive integer such that the following hold:

- For all positive integers $a$ and all $k$ less than or equal to $j,\left\langle a+\ell(j) ; r_{k}\right\rangle-\left\langle a ; r_{k}\right\rangle$ is greater than $2\left[\log s_{j-1}^{*}+3 \log s_{j}^{*}\right](j+2)$.

- For all positive integers $a$ and all $k$ less than or equal to $j,\left\langle a+\ell(j) ; r_{k}\right\rangle-\left\langle a ; r_{k}\right\rangle$ is greater than $3 p_{k}(j+2)$. 
- For all positive integers $a$, the conclusion of Lemma 7 with input values $s_{j}, M\left(s_{j}\right)$ and $n_{j}$ applies to $\left\langle a+\ell(j) ; s_{j}\right\rangle-\left\langle a ; s_{j}\right\rangle$ for $\epsilon=1 /(j+1)$ and $\delta=1 / 4$.

- For all positive integers $a$, the conclusion of Lemma 8 with input values $s_{j}, M\left(s_{j}\right)$ and $n_{j}$ applies to $\left\langle a+\ell(j) ; s_{j}\right\rangle-\left\langle a ; s_{j}\right\rangle-n_{j}$, for $\delta=1 / 4$.

- The conclusion of Lemma 13 with input values $s_{j}^{*}, U\left(s_{j}, M\left(s_{j}\right), n_{j}\right)$ as in Lemma 5 , and the set of bases $\left(\left\{r_{k}: k \leqslant j\right\} \cup\left\{r_{k}^{p_{j}}: k \leqslant j\right\}\right) \backslash\left\{s_{j}^{m}: m \in M\left(s_{j}\right)\right\}$, applies to $\ell(j)$ for $\epsilon=1 /(j+1)$ and $\delta=1 / 4$.

For $y$ an $s_{j}^{*}$-adic rational number of precision $\left(s_{j}^{*}\right)^{\left\langle a ; s_{j}^{*}\right\rangle}$, let $x(j, a, y)$ be the least number such that there is a block $w^{*}$ of length $\left\langle a+\ell(j) ; s_{j}^{*}\right\rangle-\left\langle a ; s_{j}^{*}\right\rangle$ with elements in $U\left(s_{j}, M\left(s_{j}\right), n_{j}\right)$ (as as in Lemma 5) for which the following hold. Let $w$ be the sequence in base $s_{j}$ such that $\left(w ; \ell_{U}\right)=w^{*}:$

- $x(j, a, y)=y+\left(s_{j}^{*}\right)^{-\left(\left\langle a ; s_{j}^{*}\right\rangle+1\right)} \sum_{k=0}^{\left|w^{*}\right|-1} w_{k}^{*}\left(s_{j}^{*}\right)^{-k}$.

- For all $m$ in $M\left(s_{j}\right), D\left((w ; m), \mathcal{L}\left(m, s_{j}\right)\right)<\frac{1}{j+1}$.

- $\frac{\operatorname{occ}\left(\left(w ; n_{j}\right), d_{j}\right)}{\left|\left(w ; n_{j}\right)\right|}<\frac{1}{s^{n_{j}}}-\epsilon_{j}$.

- For all $r$ in $\left(\left\{r_{k}: k \leqslant j\right\} \cup\left\{r_{k}^{p_{j}}: k \leqslant j\right\}\right) \backslash\left\{s_{j}^{m}: m \in M\left(s_{j}\right)\right\}$ and for $u$ the block of digits from position $\langle a ; r\rangle+1$ to position $\langle a+\ell(j) ; r\rangle$ in the base- $r$ expansion of $x(j, a, y)$, we have $D\left(u, B_{r}\right)<1 /(j+1)$.

We proceed by recursion on $t$ to define sequences $\left(j_{t}\right)_{t \geqslant 0},\left(b_{t}\right)_{t \geqslant 0}$ and $\left(x_{t}\right)_{t \geqslant 0}$. For $t \geqslant 0$, $j_{t}$ and $b_{t}$ are positive integers and $x_{t}$ is a $s_{j_{t}}^{*}$-adic rational number of precision $\left\langle b_{t} ; s_{j_{t}}^{*}\right\rangle$. The real $x$, which is the eventual result of our construction, will be an element of $\left[x_{t}, x_{t}+\left(s_{j_{t}}^{*}\right)^{-\left\langle b_{t} ; s_{j_{t}}^{*}\right\rangle}\right)$.

Initial stage. Let $j_{0}=0, x_{0}=0$ and $b_{0}=0$.

Stage $t+1$. Given $j_{t}, b_{t}, x_{t}$. Consider the two conditions.

1. For all bases $r \in\left\{r_{k}: k \leqslant j_{t}+1\right\} \backslash\left\{s_{j_{t}}^{m}: m \in M\left(s_{j_{t}}\right)\right\}$,

$$
\left\langle b_{t}+\left\lceil\log s_{j_{t}}^{*}+3 \log s_{j_{t}+1}^{*}\right\rceil+\ell\left(j_{t}+1\right) ; r\right\rangle-\left\langle b_{t} ; r\right\rangle<\frac{\left\langle b_{t} ; r\right\rangle}{j_{t}+1} .
$$

2. For the block $w$ composed of the first $\left\langle b_{t} ; s_{j_{t}}\right\rangle$ digits in the base- $s_{j_{t}}$ expansion of $x_{t}$,

$$
\frac{\operatorname{occ}\left(\left(w ; n_{j_{t}}\right), d_{j_{t}}\right)}{\left|\left(w ; n_{j_{t}}\right)\right|}<\frac{1}{s^{n_{j_{t}}}}-\frac{\epsilon_{j_{t}}}{2} .
$$

If both conditions hold, let $j_{t+1}=j_{t}+1$, let $a=b_{t}+\left\lceil\log s_{j_{t}}^{*}+3 \log s_{j_{t+1}}^{*}\right\rceil$ and let $y$ be the left endpoint of the leftmost $s_{j_{t+1}}^{*}$-adic subinterval of $\left[x_{t}, x_{t}+\left(s_{j_{t}}^{*}\right)^{-\left\langle b_{t} ; s_{j_{t}}^{*}\right\rangle}\right)$ of length $\left(s_{j_{t+1}}^{*}\right)^{-\left\langle a ; s_{j_{t+1}}^{*}\right\rangle}$. Otherwise, $j_{t+1}=j_{t}, a=b_{t}$, and $y=x_{t}$. Finally define,

$$
x_{t+1}=x\left(j_{t+1}, a, y\right) \text { and } b_{t+1}=a+\ell\left(j_{t+1}\right) .
$$


We check that the construction succeeds. By Lemmas 7, 8 and 13, the integer $\ell(j)$ is well defined. Indeed, in the definition of $x(j, a, y)$, each of these lemmas is applied so that at least $3 / 4$ of the blocks being considered are suitable. Thus, at least $1 / 4$ of the blocks being considered are suitable in terms of all three of the lemmas. It follows that $x(j, a, y)$ is well defined and that the sequence $x_{t}$ converges to a limit $x$.

We show that $\left(j_{t}\right)_{t \geqslant 0}$ tends to infinity with $t$. Clearly, the function $t \mapsto j_{t}$ is non-decreasing. Suppose that $\lim _{t \rightarrow \infty} j_{t}=h<\infty$ and let $t_{0}$ be such that $j_{t_{0}}=h$. By the first condition in the definition of the function $\ell$, we have $b_{t+1}>b_{t}$ for $t \geqslant 0$. Thus, since the value of $\ell\left(j_{t}\right)$ does not depend on that of $b_{t}$, there is a stage $t_{1}>t_{0}$ such that for all $t>t_{1}$ and all $r \in\left\{r_{k}: k \leqslant j_{t}\right\}$, the quantity

$$
\left\langle b_{t}+\left\lceil\log s_{h}^{*}+3 \log s_{h}^{*}\right\rceil+\ell(h) ; r\right\rangle-\left\langle b_{t} ; r\right\rangle
$$

is less than $\frac{\left\langle b_{t} ; r\right\rangle}{j_{t}+1}$. Similarly, for all stages $t>t_{0}$, we have $x_{t+1}=x\left(h, b_{t}, x_{t}\right)$. Then, by Lemma 20 for $w$ equal to the expansion of $x$ in base $s_{h}^{n_{h}}$ and the sequence of integers $\left(c_{t}\right)_{t \geqslant t_{0}}=\left(\left\langle b_{t} ; s_{h}^{*}\right\rangle\left(\ell_{U} / n_{h}\right)\right)_{t \geqslant t_{0}}$,

$$
\liminf _{t \rightarrow \infty} \frac{\operatorname{occ}\left(\left(w_{0}, \ldots, w_{c_{t}}\right), d_{h}\right)}{c_{t}+1}<\frac{1}{s_{h}^{n_{h}}}-\frac{\epsilon_{h}}{2} .
$$

Then, there must be a $t>t_{1}$ such that

$$
\frac{\operatorname{occ}\left(\left(w_{0}, \ldots, w_{c_{t}}\right), d_{h}\right)}{c_{t}+1}<\frac{1}{s_{h}^{n_{h}}}-\frac{\epsilon_{h}}{2} .
$$

For such a $t$, the criteria for defining $j_{t+1}=j_{t}+1$ are satisfied, contradicting the supposition that $\lim _{t \rightarrow \infty} j_{t}=h$. Thus, $\lim _{t \rightarrow \infty} j_{t}=\infty$.

Suppose that $s \in \mathcal{S}$ and $n \notin M(s)$. Then, there are infinitely many $j$ such that $\left(s_{j}, n_{j}\right)=(s, n)$. Fix $d$ and $\epsilon$ as guaranteed by the conclusion of Lemma 8 for $s, M(s)$ and $n$. By the previous paragraph, there are infinitely many stages $t$ such that $s=s_{j_{t}}, n=n_{j_{t}}, d=d_{j_{t}}, \epsilon=\epsilon_{j_{t}}$ and

$$
\frac{\operatorname{occ}\left(\left(w_{0}, \ldots, w_{\left\langle b_{t} ; s_{j_{t}}^{*}\right\rangle\left(\ell_{U} / n\right)}\right), d\right)}{\left\langle b_{t} ; s_{j_{t}}^{*}\right\rangle\left(\ell_{U} / n\right)+1}<\frac{1}{s^{n}}-\frac{\epsilon}{2},
$$

where $\left(w_{0}, \ldots, w_{\left\langle b_{t} ; s_{t_{t}}^{*}\right\rangle\left(\ell_{U} / n\right)}\right)$ is the sequence of digits in the base- $s^{n}$ expansion of $x_{t}$. By construction, these are also the sequence of digits in the base- $s^{n}$ expansion of $x$. Consequently, $x$ is not simply normal to base $s^{n}$.

Now, suppose that $s \in \mathcal{S}$ and $m \in M(s)$. By the definition of the sequence $\left(r_{j}\right)_{j \geqslant 0}$, fix the integer $h$ such that $s^{m}=r_{h}$. Since $\lim _{t \rightarrow \infty} j_{t}=\infty$ we can fix $t_{0}$ such that $j_{t} \geqslant h$ for all $t>t_{0}$. We consider the construction during stages $t+1>t_{0}$.

There are two possibilities for $s^{m}$ during stage $t+1$, depending on whether $s_{j_{t+1}}=s$ or not. Suppose first that $s_{j_{t+1}} \neq s$. Then, $x_{t+1}$ was chosen so that for the block $u$ of digits from position $\left\langle a ; s^{m}\right\rangle+1$ to position $\left\langle a+\ell\left(j_{t+1}\right) ; s^{m}\right\rangle=\left\langle b_{t+1} ; s^{m}\right\rangle$ in the base- $s^{m}$ expansion of $x_{t+1}$, we have $D\left(u, B_{s^{m}}\right)<1 /\left(j_{t+1}+1\right)$, where $a$ is $b_{t}$ or $b_{t}+\left\lceil\log s_{j_{t}}^{*}+3 \log s_{j_{t}+1}^{*}\right\rceil$. In the latter case, by the first condition in the definition of $\ell\left(j_{t+1}\right)$, we deduce that $\left\langle a+\ell\left(j_{t+1}\right) ; s^{m}\right\rangle-\left\langle a ; s^{m}\right\rangle$ is greater than $2\left[\log s_{j_{t}}^{*}+3 \log s_{j_{t+1}}^{*}\right\rceil\left(j_{t+1}+2\right)$. It then follows from Remark 17 that

$$
\left\langle a ; s^{m}\right\rangle-\left\langle b_{t} ; s^{m}\right\rangle<2\left\lceil\log s_{j_{t}}^{*}+3 \log s_{j_{t+1}}^{*}\right\rceil .
$$


By Lemma 18, for the block $v$ of digits in the base- $s^{m}$ expansion of $x_{t+1}$ between positions $\left\langle b_{t} ; s^{m}\right\rangle+1$ and $\left\langle b_{t+1} ; s^{m}\right\rangle$, we have $D\left(v, B_{s^{m}}\right)<2 /\left(j_{t+1}+1\right)$. By construction, we treat the base $\left(s^{m}\right)^{p_{j_{t+1}}}$ similarly during stage $t+1$ and the same conclusion applies.

Alternatively, suppose that $s=s_{j_{t}}$. Again, for the block $v$ of digits in the base- $s^{m}$ expansion of $x_{t+1}$ between positions $\left\langle b_{t} ; s^{m}\right\rangle+1$ and $\left\langle b_{t+1} ; s^{m}\right\rangle$, we have $D\left(v, B_{s^{m}}\right)<2 /\left(j_{t+1}+1\right)$ by virtue of the second condition in the definition of $x(j, a, y)$ and the above observations. Similarly, this conclusion holds for base $\left(s^{m}\right)^{p_{j t}}$. (Note, to keep the discussion simple, we have chosen to ignore the possibility of a difference between $\left\langle b_{t+1} ; s^{m}\right\rangle$ and $\left\langle b_{t+1} ; s_{U}^{\ell}\right\rangle\left(\ell_{U} / m\right)$, where $U$ is as is defined during stage $t+1$.)

Now, we consider the expansion of $x$ in base $s^{m}$. For each $t>t_{0}$, by the definition of the function $\ell$, Lemma 14 applies to the digits in this expansion from position $\left\langle b_{t} ; s^{m}\right\rangle+1$ to $\left\langle b_{t+1} ; s^{m}\right\rangle$. Thus, for each of these blocks in the expansion of $x$ in base $s^{m}$, the simple discrepancy is less than $10 /\left(j_{t+1}+1\right)$. Since $j_{t}$ tends to infinity as $t$ increases, by Lemma 19. $x$ is simply normal to base $s^{m}$.

\section{Hausdorff dimension}

Like in [13], the key tool for estimating the Hausdorff dimension of the set defined in Theorem 1 is the following result of Eggleston [6, Theorem 5].

Lemma 21. Suppose that, for $k \geqslant 1$, the set $K_{k}$ is a linear set consisting of $N_{k}$ closed intervals each of length $\delta_{k}$ and such that each interval of $K_{k}$ contains $N_{k+1} / N_{k}$ disjoint intervals of $K_{k+1}$. If $v_{0} \in(0,1]$ is such that for every $v<v_{0}$ the sum

$$
\sum_{k \geqslant 2} \frac{\delta_{k-1}}{\delta_{k}}\left(N_{k}\left(\delta_{k}\right)^{v}\right)^{-1}
$$

converges, then the Hausdorff dimension of the set $\bigcap_{k \geqslant 1} K_{k}$ is greater than or equal to $v_{0}$.

We analyze the construction of Section 3. We keep the notation from that section.

We introduce a positive real number $\eta$ such that

$$
\frac{\log \left(s_{j}^{*}-2\right)}{\log s_{j}^{*}} \geqslant \eta
$$

for $j \geqslant 0$. In view of Lemma 5 the bases $s_{j}^{*}$ may be taken arbitrarily large, thus $\eta$ can be taken arbitrarily close to 1 . For convenience, we assume that the sequence $\left(s_{j}^{*}\right)_{j \geqslant 0}$ is non-decreasing and that $s_{0}^{*} \geqslant 4$.

Let $t \geqslant 2$ be an integer. At stage $t$, by Lemmas 7,8 and 13, the number of suitable blocks $w^{*}$ is at least equal to

$$
\nu_{t}=\frac{1}{4}\left(s_{j_{t}}^{*}-2\right)^{\left\langle a+\ell\left(j_{t}\right) ; s_{j_{t}}^{*}\right\rangle-\left\langle a ; s_{j_{t}}^{*}\right\rangle},
$$

since $\# U \geqslant s_{j_{t}}^{*}-2$. Furthermore, the length of each interval is

$$
\delta_{t}=\left(s_{j_{t}}^{*}\right)^{-\left\langle b_{t} ; s_{j_{t}}^{*}\right\rangle} .
$$

Observe that

$$
\nu_{t} \geqslant \frac{1}{4}\left(s_{j_{t}}^{*}\right)^{-1} \mathrm{e}^{\eta \ell\left(j_{t}\right)} \geqslant\left(s_{j_{t}}^{*}\right)^{-2} \mathrm{e}^{\eta \ell\left(j_{t}\right)}
$$


and

$$
\left(s_{j_{t}}^{*}\right)^{-1} \mathrm{e}^{-b_{t}} \leqslant \delta_{t} \leqslant \mathrm{e}^{-b_{t}} .
$$

If $j_{t+1}=j_{t}+1$, we have in particular that

$$
\left\langle b_{t+1} ; r_{0}\right\rangle-\left\langle b_{t} ; r_{0}\right\rangle<\frac{\left\langle b_{t} ; r_{0}\right\rangle}{j_{t+1}} .
$$

This gives

$$
\mathrm{e}^{b_{t+1}-b_{t}}<r_{0}^{2} \mathrm{e}^{b_{t} / j_{t+1}}
$$

and

$$
\frac{\delta_{t}}{\delta_{t+1}} \leqslant s_{j_{t+1}}^{*} r_{0}^{2} \mathrm{e}^{b_{t} / j_{t+1}}
$$

If $j_{t+1}=j_{t}$, then we know that

$$
\left\langle b_{u+1}+\ell\left(j_{t}\right) ; r_{0}\right\rangle-\left\langle b_{u+1} ; r_{0}\right\rangle<\frac{\left\langle b_{u+1} ; r_{0}\right\rangle}{j_{t}},
$$

where $u$ is the largest integer such that $j_{u}=j_{t}-1$. As seen above, this implies that

$$
\mathrm{e}^{\ell}<r_{0}^{2} \mathrm{e}^{b_{u+1} / j_{t+1}},
$$

so, for $b \geqslant b_{u+1}$,

$$
\mathrm{e}^{b+\ell-b}<r_{0}^{2} \mathrm{e}^{b / j_{t+1}}
$$

hence we get the same upper bound on $\delta_{t} / \delta_{t+1}$ as in (4.1).

Furthermore, it is clear from the construction that

$$
\ell\left(j_{0}\right)+\ell\left(j_{1}\right)+\ldots+\ell\left(j_{t}\right) \leqslant b_{t} \leqslant \log \left(\prod_{h=0}^{j_{t}}\left(s_{h}^{*}\right)^{5}\right)+\ell\left(j_{0}\right)+\ell\left(j_{1}\right)+\ldots+\ell\left(j_{t}\right) .
$$

Also, putting $N_{t}=\nu_{1} \ldots \nu_{t}$, we have

$$
N_{t} \geqslant\left(\prod_{h=0}^{j_{t}}\left(s_{h}^{*}\right)^{-7}\right) \mathrm{e}^{\eta b_{t}} .
$$

Since the construction also ensures

$$
\left\langle 1+\ell\left(j_{t}\right) ; r_{0}\right\rangle-\left\langle 1 ; r_{0}\right\rangle \geqslant 2\left\lceil\log s_{j_{t}-1}^{*}+3 \log s_{j_{t}}^{*}\right\rceil\left(j_{t}+2\right),
$$

we get

$$
s_{j_{t}}^{*} \leqslant \mathrm{e}^{\ell\left(j_{t}\right) / 2 j_{t}} \leqslant \mathrm{e}^{b_{t} / j_{t}} .
$$

Consequently, for any positive real number $v$ and any integer $t \geqslant 2$, we obtain

$$
\frac{\delta_{t-1}}{\delta_{t}}\left(N_{t}\left(\delta_{t}\right)^{v}\right)^{-1} \leqslant\left(s_{j_{t+1}}^{*}\right)^{8} \mathrm{e}^{b_{t} / j_{t+1}} \mathrm{e}^{(v-\eta) b_{t}} \leqslant \mathrm{e}^{9 b_{t} / j_{t+1}} \mathrm{e}^{(v-\eta) b_{t}} .
$$

Since $j_{t}$ tends to infinity as $t$ increases and $\left(b_{t}\right)_{t \geqslant 0}$ is a strictly increasing sequence of integers, the corresponding series converges for every $v<\eta$. It then follows from Lemma 21 that the dimension of the set into consideration is not less than $\eta$. Recalling that $\eta$ can be taken arbitrarily close to 1, this proves the last assertion of Theorem 1. 


\section{References}

[1] Verónica Becher and Theodore A. Slaman. On the normality of numbers to different bases. preprint, 2013.

[2] Émile Borel. Les probabilités dénombrables et leurs applications arithmétiques. Supplemento Rendiconti del Circolo Matematico di Palermo, 27:247-271, 1909.

[3] Yann Bugeaud. Distribution modulo one and Diophantine approximation, volume 193 of Cambridge Tracts in Mathematics. Cambridge University Press, Cambridge, 2012.

[4] Yann Bugeaud. On the expansions of a real number to several integer bases. Revista Matemática Iberoamericana, 28(4):931-946, 2012.

[5] J. W. S. Cassels. On a problem of Steinhaus about normal numbers. Colloquium Mathematicum, 7:95-101, 1959.

[6] H. G. Eggleston. Sets of fractional dimensions which occur in some problems of number theory. Proceedings of the London Mathematical Society. Second Series, 54:42-93, 1952.

[7] Mark Haiman, 2013. Private correspondence.

[8] G. H. Hardy and E. M. Wright. An introduction to the theory of numbers. Oxford University Press, Oxford, sixth edition, 2008.

[9] Peter Hertling. Simply normal numbers to different bases. Journal of Univesal Computer Science, 8(2):235-242 (electronic), 2002.

[10] L. Kuipers and H. Niederreiter. Uniform distribution of sequences. Dover, 2006.

[11] Calvin T. Long. Note on normal numbers. Pacific Journal of Mathematics, 7:1163-1165, 1957.

[12] John E. Maxfield. Normal k-tuples. Pacific Journal of Mathematics, 3:189-196, 1953.

[13] A. D. Pollington. The Hausdorff dimension of a set of normal numbers. Pacific Journal of Mathematics, 95(1):193-204, 1981.

[14] Wolfgang M. Schmidt. On normal numbers. Pacific Journal of Mathematics, 10:661-672, 1960.

[15] Wolfgang M. Schmidt. Über die Normalität von Zahlen zu verschiedenen Basen. Acta Arithmetica, 7:299-309, 1961/1962. 